\title{
KILLING VECTOR FIELDS AND HOLONOMY ALGEBRAS
}

\author{
CARLOS CURRÁS-BOSCH
}

\begin{abstract}
We prove that for each Killing vector field $X$ on a complete Riemannian manifold, whose orthogonal distribution is involutive, the $(1,1)$ skew-symmetric operator $A_{X}$ associated to $X$ by $A_{X}=L_{X}-\nabla_{X}$ lies in the holonomy algebra at each point. By using the same techniques, we also study when that operator lies in the infinitesimal and local holonomy algebras respectively.
\end{abstract}

Introduction. Kostant (see [2 or 3]) proved that if $X$ is a Killing vector field on a compact Riemannian manifold $M$, for each point $x$ of $M,\left(A_{X}\right)_{x}$ lies in the holonomy algebra $\mathfrak{B}(x), A_{X}$ is associated to $X$ by $A_{X}=L_{X}-\nabla_{X}$ ( $L$ is the Lie derivative and $\nabla$ the Riemannian connection). The proof is based on a decomposition, $A_{X}=S_{X}+B_{X}$, where $\left(S_{X}\right)_{x} \in \mathscr{S}(x)$ and $\left(B_{X}\right)_{x} \in \mathscr{S}(x)^{\perp}$, where $\mathscr{S}(x)^{\perp}$ is the orthogonal complement of $\mathbb{S}(x)$ in the algebra $E(x)$ of skew-symmetirc endomorphisms of $T_{x}(M)$, with respect to the inner product given by $(A, B)=-\operatorname{trace}(A \circ B)$. One observes that $B_{X}$ is parallel and as $\operatorname{div}\left(B_{X} X\right)=-\operatorname{trace}\left(B_{X}^{2}\right)$, if $M$ is compact it results in $B_{X}=0$.

In this paper we are concerned with noncompact and complete Riemannian manifolds and a Killing vector field $X$ whose orthogonal distribution is involutive. We prove in $\S 2$ that $\left(A_{X}\right)_{x} \in \mathscr{S}(x), \forall x \in M$. We apply the techniques used in this proof to study in $\S 3$ when $\left(A_{X}\right)_{x}$ lies in $\mathscr{S}^{\prime}(x)$ and $\mathbb{S}^{*}(x)$ (infinitesimal and local holonomy algebras respectively).

1. Properties of some distributions. Throughout this paper we suppose that $M$ is a complete, connected Riemannian manifold of dimension $n+1$, with metric $g$ and Riemannian connection $\nabla$. We set $w=i_{X} g$, where $X$ is a Killing vector field.

LEMMA 1.1. $d w(Y, Z)=-g\left(A_{X} Y, Z\right)$.

Proof.

$$
\begin{aligned}
2 d w(Y, Z)= & Y(w(Z))-Z(w(Y))-w([Y, Z]) \\
= & Y(g(X, Z))-Z(g(X, Y))-g(X,[Y, Z]) \\
= & g\left(\nabla_{Y} X, Z\right)+g\left(X, \nabla_{Y} Z\right)-g\left(\nabla_{Z} X, Y\right) \\
& -g\left(X, \nabla_{Z} Y\right)-g\left(X, \nabla_{Y} Z-\nabla_{Z} Y\right) \\
= & g\left(\nabla_{v} X, Z\right)-g\left(\nabla_{Z} X, Y\right) .
\end{aligned}
$$

Received by the editors July 2, 1982 and, in revised form, April 6, 1983. Partially presented in September 1981 to "VI Congrès du Groupement des Mathématiciens d'Expression Latine".

1980 Mathematics Subject Classification. Primary 53C20.

Key words and phrases. Complete Riemannian manifolds, Killing vector fields, holonomy algebras. 
As $A_{X} U=-\nabla_{U} X$ for each vector field $U$, and $A_{X}$ is skew-symmetric, we have $2 d w(Y, Z)=-2 g\left(A_{X} Y, Z\right)$.

We assume that the orthogonal distribution to $X$ is involutive; i.e., there exists a 1 -form $\phi$ such that $d w=\phi \wedge w$.

We can assume that $d w$ is nowhere zero because if $\left(A_{X}\right)_{x}=0$, at some point $x$ of $M,\left(B_{X}\right)_{x}=0$ and as $B_{X}$ is parallel, $B_{X}=0$.

We also assume that $X$ is nowhere zero, because we consider its orthogonal distribution, but as is well known, if a vector bundle has a nowhere zero crosssection then its Euler class must be zero (see [1, Theorem 8.3, p. 242]). So from now on, the manifolds to consider must have Euler class zero.

Let $\mathcal{P}$ be the involutive orthogonal distribution to $X$, and let $\left\{Z_{1}, \ldots, Z_{n}\right\}$ be a local orthonormal basis of $\mathcal{P}$ near $x$, so that throughout that neighborhood $d w=\lambda z^{1}$ $\wedge w$, where $\left\{(1 /\|X\|) w ; z^{1}, \ldots, z^{n}\right\}$ is the dual basis of $\left\{(1 /\|X\|) X ; Z_{1}, \ldots, Z_{n}\right\}$.

In such a basis the matrix of $A_{X}$ takes the form

$$
\left[\begin{array}{ccccc}
0 & -\lambda\|X\| & 0 & \ldots & 0 \\
\lambda\|X\| & 0 & . & \cdots & 0 \\
0 & 0 & . & \ldots & \cdot \\
. & . & . & \cdots & \cdot \\
0 & 0 & . & \cdots & 0
\end{array}\right] .
$$

From $A_{X}$ we know all the curvature transformations $R\left(X, Z_{i}\right)(1 \leqslant i \leqslant n)$ because $R\left(X, Z_{i}\right)=\nabla_{Z_{i}} A_{X}$

We can observe

LEMMA 1.2. The integral manifolds of $\mathcal{P}$ are all totally geodesic.

Proof. $g\left(\nabla_{Z_{i}} Z_{j}, X\right)=-g\left(Z_{j}, \nabla_{Z_{i}} X\right)=g\left(Z_{j}, A_{X} Z_{i}\right)=0(1 \leqslant i, j \leqslant n)$.

LEMMA 1.3. $\left\{Z_{2}, \ldots, Z_{n}\right\}$ and $\left\{X, Z_{1}\right\}$ are involutive distributions.

Proof. Consider the indices $\alpha, \beta ; 2 \leqslant \alpha, \beta \leqslant n$. We already know that

$$
g\left(X, \nabla_{Z_{\alpha}} Z_{\beta}\right)=0 \text {. }
$$

Now,

$$
\begin{aligned}
\lambda\|X\|^{2} g\left(Z_{1}, \nabla_{Z_{\alpha}} Z_{\beta}\right) & =g\left(A_{X} X, \nabla_{Z_{\alpha}} Z_{\beta}\right)=-g\left(\nabla_{Z_{\alpha}}\left(A_{X} X\right), Z_{\beta}\right) \\
& =-g\left(\left(\nabla_{Z_{\alpha}} A_{X}\right)(X), Z_{\beta}\right)-g\left(A_{X}\left(\nabla_{Z_{\alpha}} X\right), Z_{\beta}\right) \\
& =-g\left(R\left(X, Z_{\alpha}\right) X, Z_{\beta}\right) .
\end{aligned}
$$

So we have

(1) $g\left(A_{X} X, \nabla_{Z_{\alpha}} Z_{\beta}\right)=-g\left(R\left(X, Z_{\alpha}\right) X, Z_{\beta}\right)$ and

(2) $g\left(A_{X} X, \nabla_{Z_{\beta}} Z_{\alpha}\right)=-g\left(R\left(X, Z_{\beta}\right) X, Z_{\alpha}\right)$.

Subtracting (2) from (1) gives us $g\left(A_{X} X,\left[Z_{\alpha}, Z_{\beta}\right]\right)=0$.

To prove that $\left\{X, Z_{1}\right\}$ is involutive, we consider

$$
L_{X}\left(A_{X} X\right)=L_{X}\left(\lambda\|X\|^{2} Z_{1}\right)=X\left(\lambda\|X\|^{2}\right) Z_{1}+\lambda\|X\|^{2} L_{X} Z_{1}=0
$$

because $L_{X}\left(A_{X} X\right)=L_{X}\left(-\nabla_{X} X\right)=0$, so $L_{X} Z_{1}=0$. 
It is well known (see [2, Corollary 4.3, p. 246]) that if $X$ is an infinitesimal affine transformation, then at each $x \in M,\left(A_{X}\right)_{x}$ belongs to the normaliser of $\mathscr{S}(x)$, $N(\mathbb{S}(x))$. The same result is true for $\mathbb{S}^{\prime}(x)$ as can be proved easily, using that (S) $^{\prime}(x)$ is spanned by all linear endomorphisms of $T_{x}(M)$ of the form $\left(\nabla^{k} R\right)\left(Y, Z ; V_{1}, \ldots, V_{k}\right)$, where $Y, Z ; V_{1}, \ldots, V_{k} \in T_{x}(M)$ and $0 \leqslant k<\infty$.

It is not difficult to give some examples of Riemannian manifolds with a Killing vector field $X$, such that the condition $d w=\phi \wedge w$ is verified. We give here three examples such that:

(i) the orbits of $X$ are all diffeomorphic to $R$;

(ii) the orbits of $X$ are all diffeomorphic to $S^{1}$;

(iii) the orbits of $X$ are solenoids on a torus.

(i) Let $\left(N, g^{\prime}\right)$ be a complete Riemannian manifold. We consider $R \times N$. Let $t$ be the parameter in $R$ given by the identity map and $X=d / d t$.

Take in $R \times N$ the Riemannian metric $g$ such that its restriction to $T(N)$ is $g^{\prime}, X$ is orthogonal to $T(N)$ at all points and $\|X\|$ is given by a definite positive function on $N$. Take $w_{X}=i_{X} g$. It is verified that $d w_{X}=\phi \wedge w_{X}$ and the vector field $Z_{1}$ considered above is $\gamma \cdot \operatorname{grad}(\|X\|)$.

(ii) Taking $S^{1}$ instead of $R$, by the same argument, we obtain a Killing vector field with $d w=\phi \wedge w$, and now the orbits are all diffeomorphic to $S^{1}$.

(iii) Taking $S^{1} \times S^{1} \times N$, we associate $Y$ to the first $S^{1}$ factor and $Z$ to the second $S^{1}$ factor. We define $g$ such that $g(Y, Z)=0$ and $g(Y, Y)=g(Z, Z)$ is given by a definite positive function on $N ; Y$ and $Z$ are orthogonal to $T(N)$, and $g$ restricted to $T(N)$ is $g^{\prime}$. Now it is verified that $d w_{Y}=\phi \wedge w_{Y}, d w_{Z}=\phi \wedge w_{Z}, \phi$ is the same for $w_{Y}$ and $w_{Z}$; so the same condition is verified considering $X=\alpha Y+\beta Z, \alpha$ and $\beta$ real numbers; then taking $\alpha$ and $\beta$ such that $\alpha / \beta \notin Q$, it is verified that $d w_{X}=\phi \wedge w_{X}$ and the orbits of $X$ are solenoids on a torus.

It should be pointed out that these three examples give us the three possible types of orbit for Killing vector fields on noncompact manifolds (take $N$ noncompact in (ii) and (iii)).

2. $\left(A_{X}\right)_{x}$ belongs to $\mathbb{S}(x)$. We calculate $R\left(X, Z_{i}\right)(1 \leqslant i \leqslant n)$. We begin by the following observations.

$A_{X} X=\lambda\|X\|^{2} Z_{1}$, as we proved in $\S 1$, so $\nabla_{X} X=-\lambda\|X\|^{2} Z_{1}$, and $\nabla_{X}((1 /\|X\|) X)$ $=(1 /\|X\|) \nabla_{X} X=-\lambda\|X\| Z_{1}$.

As the integral manifolds of $\mathcal{P}$ are totally geodesic, $\nabla_{Z_{i}}((1 /\|X\|) X)=0$, so for $i=1$,

$$
\begin{aligned}
\nabla_{Z_{1}}((1 /\|X\|) X) & =Z_{1}(1 /\|X\|) X+(1 /\|X\|) \nabla_{Z_{1}} X \\
& =Z_{1}(1 /\|X\|) X-(1 /\|X\|) A_{X} Z_{1} \\
& =Z_{1}(1 /\|X\|) X+(1 /\|X\|) \lambda X=0 .
\end{aligned}
$$

For $Z_{\alpha}, 2 \leqslant \alpha \leqslant n$, we have

$$
\nabla_{Z_{\alpha}}((1 /\|X\|) X)=0 \text {, so } Z_{\alpha}(\|X\|)=0 .
$$


As $R\left(X, Z_{i}\right)=\nabla_{Z_{i}} A_{X}$, in the basis $\left\{(1 /\|X\|) X, Z_{1}, \ldots, Z_{n}\right\}$, we obtain

$$
R\left(X, Z_{i}\right)=\left[\begin{array}{ccccc}
0 & -Z_{i}(\lambda\|X\|) & -\lambda\|X\| \Gamma_{i 1}^{2} & \ldots & -\lambda\|X\| \Gamma_{i 1}^{n} \\
Z_{i}(\lambda\|X\|) & 0 & 0 & \cdots & 0 \\
\lambda\|X\| \Gamma_{i 1}^{2} & 0 & 0 & \cdots & 0 \\
\cdot & \cdot & \cdot & \cdots & \cdot \\
\cdot & \cdot & \cdot & \cdots & \cdot \\
\cdot & \cdot & \cdot & \cdots & . \\
\lambda\|X\| \Gamma_{i 1}^{n} & 0 & 0 & \cdots & 0
\end{array}\right] .
$$

In order to simplify the notations, we let

$$
\eta=\left[\begin{array}{ccccc}
0 & -a & -a_{2} & \ldots & -a_{n} \\
a & 0 & 0 & \ldots & 0 \\
a_{2} & 0 & 0 & \ldots & 0 \\
\cdot & . & . & \ldots & . \\
. & . & . & \ldots & . \\
. & . & . & \ldots & . \\
a_{n} & 0 & 0 & \ldots & 0
\end{array}\right], \quad \kappa=\left[\begin{array}{ccccc}
0 & -b & 0 & \ldots & 0 \\
b & 0 & 0 & \ldots & 0 \\
0 & 0 & 0 & \ldots & 0 \\
\cdot & . & . & \ldots & . \\
\cdot & . & . & \ldots & . \\
. & . & . & \ldots & . \\
0 & 0 & 0 & \ldots & 0
\end{array}\right] .
$$

$R\left(X, Z_{i}\right)$ is of the form $\eta$ and belongs to $\mathscr{S}(x)$, while $A_{X}$ is of the form $\kappa$ and belongs to $N(\mathbb{S}(x))$.

We calculate the elements of $\mathscr{S}(x),[\kappa, \eta]$ and $[\kappa,[\kappa, \eta]]$.

$$
\begin{aligned}
& {[\kappa, \eta]=\left[\begin{array}{ccccc}
0 & 0 & 0 & \ldots & 0 \\
0 & 0 & -b a_{2} & \ldots & -b a_{n} \\
0 & b a_{2} & 0 & \ldots & 0 \\
\cdot & \cdot & . & \ldots & \cdot \\
\cdot & . & . & \ldots & . \\
\cdot & \cdot & . & \ldots & \cdot \\
0 & b a_{n} & 0 & \ldots & 0
\end{array}\right]} \\
& {[\kappa,[\kappa, \eta]]=\left[\begin{array}{ccccc}
0 & 0 & b^{2} a_{2} & \ldots & b^{2} a_{n} \\
0 & 0 & 0 & \ldots & 0 \\
-b^{2} a_{2} & 0 & 0 & \ldots & 0 \\
\cdot & . & . & \ldots & . \\
\cdot & . & . & \ldots & . \\
\cdot & . & . & \ldots & . \\
-b^{2} a_{n} & 0 & 0 & \ldots & 0
\end{array}\right] .}
\end{aligned}
$$

If $a \neq 0$, there exist real numbers $A, B$ such that $\eta=A \kappa+\left(1 / B^{2}\right)[\kappa,[\kappa, \eta]]$, so $\kappa=(1 / A)\left(\eta-\left(1 / B^{2}\right)[\kappa,[\kappa, \eta]]\right)$, whence the right side clearly belongs in $\mathbb{S}(x)$. 
If $a=0$ and at least one of $a_{2}, \ldots, a_{n}$ is $\neq 0$, we calculate $[\eta,[\kappa, \eta]] \in \mathscr{S}(x)$ and obtain

$$
[\eta,[\kappa, \eta]]=\left[\begin{array}{ccccc}
0 & -b\left(a_{2}^{2}+\cdots+a_{n}^{2}\right) & 0 & \cdots & 0 \\
b\left(a_{2}^{2}+\cdots+a_{n}^{2}\right) & 0 & 0 & \cdots & 0 \\
0 & 0 & 0 & \cdots & 0 \\
\cdot & \cdot & . & \cdots & . \\
\cdot & \cdot & . & \cdots & . \\
\cdot & . & . & \cdots & . \\
0 & 0 & 0 & \cdots & 0
\end{array}\right] .
$$

Therefore, there exists a real number $C$ such that $\kappa=C[\eta,[\kappa, \eta]]$.

If $a=a_{2}=\cdots=a_{n}=0$, then this means that at all points of $M, i_{X} R=0$. Hence $\Gamma_{11}^{\alpha}=\Gamma_{\alpha \beta}^{1}=0$, for all $\alpha, \beta \in\{2, \ldots, n\}$. In this case the distributions $\left\{X, Z_{1}\right\}$ and $\left\{Z_{2}, \ldots, Z_{n}\right\}$ are parallel, so that the elements of $(S)(x)$ are of the form

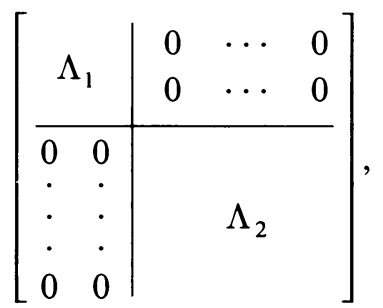

where $\Lambda_{1}$ is a $2 \times 2$ matrix and $\Lambda_{2}$ is $(n-1) \times(n-1)$.

At each point $x, R\left(X, Z_{1}\right)=0, \Lambda_{1}=0$; so, $\left(A_{X}\right)_{x} \in(\circlearrowleft(x))^{\perp}$ and $A_{X}=B_{X}$.

In this case $\psi_{x}^{0}$ (the restricted holonomy group) is reducible and $T_{x}(M)$ has an invariant subspace on which $\psi_{x}^{0}$ acts trivially, of dimension $\geqslant 2$, which contains $X$ at all points. Let $\tilde{M}$ be the universal covering manifold of $M$ with the induced Riemannian metric. The holonomy group of $\tilde{M}$ at $\tilde{x}(\pi(\tilde{x})=x)$ is $\psi_{x}^{0}$ and by the De Rham Decomposition Theorem, we have $\tilde{M}=R^{2+q} \times N$, where in the $R^{2+q}$ factor we have the Killing vector field induced by $X$, which we also represent by $X$, such that $d w=\phi \wedge w$. But this cannot occur in $R^{2+q}$, unless $X$ is parallel and $A_{X}=0$.

So we have

THEOREM 2.1. Let $(M, g)$ be a complete and connected Riemannian manifold. Let $X$ be a Killing vector field on $M$, such that $d w=\phi \wedge w$, for $w=i_{X} g$. Then, $\left(A_{X}\right)_{x} \in$ (S) $(x)$, for all $x$ in $M$.

3. Local and infinitesimal holonomy algebras. Throughout this section we continue to assume that $d w=\phi \wedge w$.

LEMMA 3.1. If $\left(i_{X} R\right)_{x} \neq 0,\left(A_{X}\right)_{x} \in \mathfrak{S S}^{\prime}(x)$.

Proof. There exists some $i, 1 \leqslant i \leqslant n$, such that $R\left(X, Z_{i}\right) \neq 0$, so in (S)'(x) there is some element of the form $\eta$, and as $\left(A_{X}\right)_{x}$ is of the form $\kappa \in N\left(\mathcal{S}^{\prime}(x)\right)$, by using the same method as in $\$ 2$, we obtain $\left(A_{X}\right)_{x} \in \mathbb{S S}^{\prime}(x)$. 
We study which of the above mentioned holonomy algebras contains $A_{X}$.

We can assume that $A_{X} \neq 0$, so $X \neq 0$, because we have supposed $d w=\phi \wedge w$.

Let $U=\left\{x \in M \mid\left(i_{X} R\right)_{x} \neq 0\right\}$. $U$ is an open set and if $x \in M \circ U$, there exists an open neighborhood of $x, V_{x}$, where $i_{X} R \neq 0$. In $V_{x},\left\{X, Z_{1}\right\}$ and $\left\{Z_{2}, \ldots, Z_{n}\right\}$ are parallel distributions and, in the same way as in $\S 2$, we can prove that $\left(A_{X}\right)_{x} \in$ $\left((S *(x))^{\perp}, \psi^{*}(x)\right.$ (the local holonomy group) is reducible and $T_{x}(M)$ has an invariant subspace on which $\psi^{*}(x)$ acts trivially, of dimension $\geqslant 2$.

If $x \in \partial(U)$, the boundary of $U$, we consider an open neighborhood of $x, W_{x}$, such that for each $y \in W_{x}$ and the parallel transport $\tau$ along any curve in $W_{x}$ from $x$ to $y$, we have $\tau^{-1}\left(S^{*}(y) \tau \subset \mathbb{S} *(x)\right.$. Such $W_{x}$ exists by Proposition 10.1, Chapter II of [2].

Let $\left\{y_{n}\right\}$ be a sequence $\left\{y_{n}\right\} \rightarrow x$ and $y_{n} \in W_{x} \cap U, \forall n$; we have $\left(A_{X}\right)_{y_{n}} \in\left(\mathcal{S S}^{\prime}\left(y_{n}\right)\right.$ $\subset$ SS* $\left(y_{n}\right)$. Let $\tau_{n}$ be the parallel transport along the minimizing geodesic from $x$ to $y_{n}$ (we can take $W_{x}$ convex), then $\tau_{n}^{-1} \mathscr{S S}^{*}\left(y_{n}\right) \tau_{n} \subset \mathcal{S S}^{*}(x)$, and as $\left(A_{X}\right)_{y_{n}} \in \mathcal{S S}^{\prime}\left(y_{n}\right) \subset$ (S) $\left(y_{n}\right)$, we obtain $\tau_{n}^{-1}\left(A_{X}\right)_{y_{n}} \tau_{n} \in \mathbb{B S F}^{*}(x)$; so $\left(A_{X}\right)_{x} \in$ SF $^{*}(x)$.

We have proved

THEOREM 3.1. There exists an open set $U$, defined by $U=\left\{x \in M \mid\left(i_{X} R\right)_{x} \neq 0\right\}$, such that:

(a) if $x \in U,\left(A_{X}\right)_{x} \in \mathbb{S S}^{\prime}(x)$,

(b) if $x \in M \circ U,\left(A_{X}\right)_{x} \in\left(\mathbb{S S}^{*}(x)\right)^{\perp}$,

(c) if $x \in \partial(U),\left(A_{X}\right)_{x} \in \mathbb{B S}^{*}(x)$.

\section{REFERENCES}

1. D. Husemoller, Fibre bundles, 2nd ed., Graduate Texts in Math., Vol. 20, Springer-Verlag, Berlin and New York, 1975.

2. S. Kobayashi and K. Nomizu, Foundations of differential geometry, Vol. I, Interscience, New York, 1963.

3. B. Kostant, The holonomy and the Lie algebra of infinitesimal motions of a Riemannian manifold, Trans. Amer. Math. Soc. 80 (1955), 528-542.

Departamento Geometria y Topologia, Facultad Matemáticas, Universidad de Barcelona, Barcelona, Spain 